\title{
Blockade of the Dioxin Pathway by Herbal Medicine Formula Bupleuri Minor: Identification of Active Entities for Suppression of AhR Activation
}

\author{
Ayumi Kasai, Nobuhiko Hiramatsu, Kunihiro Hayakawa, Jian Yao, and Masanori Kitamura* \\ Department of Molecular Signaling, Interdisciplinary Graduate School of Medicine and Engineering, University of \\ Yamanashi; Shimokato 1110, Chuo, Yamanashi 409-3898, Japan. \\ Received December 21, 2007; accepted February 21, 2008; published online February 25, 2008
}

\begin{abstract}
Environmental pollutants including dioxins activate the aryl hydrocarbon receptor (AhR) and cause a wide range of pathologies. Development of AhR antagonists will be useful for prevention and treatment of the diseases related to AhR activation. Towards this goal, we aimed at seeking for potential AhR antagonists in herbal medicines using the dioxin responsive element-based sensing via secreted alkaline phosphatase (DRESSA). Through initial rough screening, 4 formulae were selected from 20 herbal medicines and subjected to the second, detailed screening. We found that only Formula bupleuri minor (TJ-9) significantly inhibited activation of AhR by 2,3,7,8tetrachlorodibenzo-p-dioxin. Among 7 raw herb extracts in TJ-9, Glycyrrhizae Radix and Scutellariae Radix were responsible for the antagonistic effect of TJ-9 against dioxin. Some constituents including Bupleuri Radix and Zingiberis Rhizoma rather activated AhR. Among 12 major constituents of Glycyrrhizae Radix and Scutellariae Radix, we identified that licopyranocoumarin, glycyrrhizic acid and genistein in Glycyrrhizae Radix and baicalein, wogonin and daidzein in Scutellariae Radix had substantial antagonistic effects on AhR. Among these, baicalein most effectively blocked activation of $A h R$ triggered by cigarette smoke, a strong activator of $A h R$. The antagonistic substances identified here may be useful for prevention from diseases associated with aberrant activation of AhR.
\end{abstract}

Key words herbal medicine; dioxin; aryl hydrocarbon receptor; secreted alkaline phosphatase

In the daily life, we are exposed to a wide range of environmental pollutants. In particular, dioxins and dioxin-like chemicals are contained in foods and polluted air, and these toxic substances are taken mainly through ingestion and respiration. Cigarette smoke also contains high levels of dioxinlike pollutants that trigger the similar signaling pathway to that of dioxins in vitro and in vivo. ${ }^{1)}$

Dioxins and dioxin-like substances cause a diverse range of species- and tissue-specific toxicities including carcinogenicity, immunotoxicity, endocrine dysregulation, and reproductive/developmental abnormalities. ${ }^{2,3)}$ Previous reports showed that toxic effects of these pollutants are mediated by the aryl hydrocarbon receptor (AhR), a ligand-dependent transcription factor. ${ }^{4)}$ When cells are exposed to these toxic substances, the toxicants pass through the cell membrane and bind to the cytosolic AhR. Subsequently, the AhR-ligands translocate into the nucleus and heterodimerize with the AhR nuclear translocator. These complexes then bind to the xenobiotic responsive element [XRE, also called dioxin responsive element (DRE)], leading to transcriptional induction of dioxin-responsive genes including cytochrome P450 1A1 (CYP1A1). ${ }^{5)}$ Previous investigation using AhR-null mutant mice confirmed a critical role of AhR in the toxic effects of dioxins. ${ }^{6}$ Furthermore, constitutive activation of AhR via genetic manipulation caused malignant tumors and immune abnormality in mice even without exposure to xenobiotic ligands. ${ }^{7,8)}$ Savouret et al. also indicated that activation of AhR by xenobiotic ligands may be a fundamental trigger for cardiovascular diseases. ${ }^{9)}$ Of note, cigarette smoke, a strong activator of $A h R,{ }^{1)}$ is one of the most famous risk factors for cancers and cardiovascular diseases. Based on this current concept and knowledge, development of $\mathrm{AhR}$ antagonists is important for prevention and treatment of AhR-associated diseases caused by dioxins and other xenobiotic ligands.

As described, we recently reported that cigarette smoke contains high levels of agonists for AhR using a cell-based reporter assay. Using reporter transgenic mice, we also provided in vivo evidence that exposure of the mice to cigarette smoke causes significant, sustained activation of AhR. ${ }^{1}$ Cigarette smoke is known to cause a variety of pathologies including cancers, obstructive pulmonary diseases, atherosclerosis, cardiovascular/cerebrovascular diseases and developmental/reproductive abnormalities. ${ }^{10)}$ Many of these pathologies are also caused by dioxins and dioxin-like chemicals. ${ }^{3,9)}$ The fact that cigarette smoke exhibited high levels of the dioxin-like potential for AhR activation suggested a possibility that the health problems known in smokers are, at least in part, due to the potential of cigarette smoke to activate the AhR-XRE/DRE pathway. Development of AhR antagonists may, therefore, also be useful for prevention of cigarette smoke-related disorders in active and passive smokers. Towards this goal, we aimed in the present study at seeking for AhR antagonists in herbal medicines. Herbal medicines were used as initial sources because; 1) biologically active substances may be enriched in herbal medicines during the longterm clinical experiences, and 2) chemical constituents in individual herb extracts have been analyzed in detail by modern pharmaceutical industries, which should be useful for identification of active entities.

For the screening of AhR antagonists, we used the DREbased sensing via secreted alkaline phosphatase (DRESSA) bioassay. It is a fast, sensitive and economical assay that can detect and quantify activation of AhR by dioxins, dioxin-like chemicals and other xenobiotic compounds. ${ }^{11-13)}$ In this approach, a murine hepatoma cell line Hepa-1c1c7 was stably transfected with a secreted alkaline phosphatase (SEAP) gene under the control of the XRE/DRE consensus sequences. The established sensor clones secreted SEAP following stimulation with 2,3,7,8-tetrachlorodibenzo-p-dioxin $(2,3,7,8-\mathrm{TCDD})$ in a dose-dependent manner. Secretion of 
SEAP was also induced by other activators of AhR (3methylcholanthrene, benzo[ $a]$ pyrene, $\beta$-naphthoflavone), but not by other unrelated stimuli. ${ }^{11)}$ DRESSA assay can be performed easily in mini-scales using 96- or 384-well plates, and the whole assay (from cell seeding to chemiluminescence assay) can be completed within several hours. ${ }^{12)} \mathrm{Be}-$ cause of these properties, DRESSA is advantageous for highthroughput screening of chemicals that affect the AhRXRE/DRE pathway.

Using this approach, we here demonstrate that, among 20 herbal medicines tested, Formula bupleuri minor (Sho-saikoto; TJ-9) significantly inhibited activation of AhR by 2,3,7,8TCDD. Among 7 raw herb extracts contained in TJ-9, Glycyrrhizae Radix and Scutellariae Radix were responsible for its antagonistic effect. We also provide evidence that licopyranocoumarin, glycyrrhizic acid and genistein in Glycyrrhizae Radix and baicalein, wogonin and daidzein in Scutellariae Radix had substantial antagonistic effects on AhR. Among these substances, we further demonstrate that baicalein, but not others, significantly blocked activation of AhR triggered by cigarette smoke.

\section{MATERIALS AND METHODS}

Reagents Herbal medicine extracts (TJ-7, TJ-9, TJ-10, TJ-12, TJ-18, TJ-23, TJ-36, TJ-41, TJ-43, TJ-47, TJ-48, TJ53, TJ-54, TJ-62, TJ-83, TJ-87, TJ-100, TJ-107, TJ-108, TJ114), raw herb extracts (Bupleuri Radix, Pinelliae Tuber, Scutellariae Radix, Zizyphi Fructus, Ginseng Radix, Glycyrrhizae Radix, Zingiberis Rhizoma) and their constituents (glycycoumarin, isoliquiritin, licopyranocoumarin, liquiritin apioside, skullcapflavone II) were kindly provided by Tsumura \& Co. (Tokyo, Japan). Baicalein, baicalin, genistein, glycyrrhizic acid, liquiritin, wogonin and 2,3,7,8-TCDD were purchased from Wako Pure Chemical Industries Ltd. (Osaka, Japan), daidzein was from Fujicco (Kobe, Japan) and $\alpha$-naphthoflavone $(\alpha \mathrm{NF})$ was obtained from Sigma-Aldrich Japan (Tokyo, Japan). All chemicals were dissolved in dimethyl sulfoxide (DMSO), except for initial screening of herbal medicine extracts that were dissolved in phosphate buffered saline (PBS).

Preparation of Cigarette Smoke Extract (CSE) A Japanese major cigarette brand (tar content: $10 \mathrm{mg}$ ) was used for studies. CSE was prepared as described before. ${ }^{1)}$ In brief, following the method of International Organization for Standardization, mainstream smoke was made using constant vacuum flow (10.5 1/min) and collected in a glass bottle (volume: 21$)$ containing PBS. The smoke from one cigarette was dissolved in $100 \mathrm{ml}$ PBS with vigorous shaking for $5 \mathrm{~min}$, and CSE was prepared. This material was considered as $100 \%$ CSE. Four lots of CSE were independently prepared and stored at $-20^{\circ} \mathrm{C}$ until use.

Cells Murine and human hepatoma cell lines Hepa1c1c7 and HepG2 were purchased from American Type Culture Collection (Manassas, VA, U.S.A.). HeXS34 (Hepa-

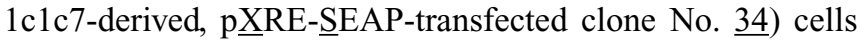
were established by transfection of Hepa-1c1c7 cells with pXRE-SEAP that introduces a SEAP gene under the control of 2 copies of the XRE consensus sequence, as described previously. ${ }^{13)}$ HeSS8 (Hepa-1c1c7-derived, p్V40-SEEAPtransfected clone No. $\underline{8}$ ) cells were also established by trans- fection of Hepa-1c1c7 cells with pSV40-SEAP that introduces a SEAP gene under the control of the simian virus 40 (SV40) promoter. ${ }^{1)}$ HeSS8 cells constitutively secreting SEAP were used as a control for the DRESSA assay. Cells were maintained in $\alpha$-minimum essential medium ( $\alpha$-MEM; Invitrogen, Carlsbad, CA, U.S.A.) supplemented with $5 \%$ fetal bovine serum (FBS).

Pharmacological Treatments HeXS34 cells and HeSS8 cells were seeded in 96-well plates (10000 cells/well) and incubated for $24 \mathrm{~h}$. The cells were pretreated with herbal medicine extracts, raw herb extracts or individual chemicals at various concentrations for $30 \mathrm{~min}$, and subsequently treated with $2,3,7,8$-TCDD (25 pM) or CSE (1\%) in the presence of test agents for $24 \mathrm{~h}$. After the treatments, culture media and cells were subjected to; i) chemiluminescent assay to evaluate SEAP activity and ii) formazan assay to assess cell viability, as described later. $\alpha$-MEM containing $1 \%$ FBS was generally used for experiments. In all experiments, the concentration of DMSO was kept at less than $0.3 \%$.

SEAP Assay Activity of SEAP in culture media was evaluated by a chemiluminescent method using Great EscAPe SEAP detection kit (BD Bioscience, Palo Alto, CA, U.S.A.), as described before. ${ }^{14)}$ In brief, $5 \mu$ l of culture media were mixed with $15 \mu \mathrm{l}$ of $1 \times$ dilution buffer and incubated for $30 \mathrm{~min}$ at $65^{\circ} \mathrm{C}$. After the incubation, samples were mixed with $20 \mu \mathrm{l}$ of assay buffer containing L-homoarginine, left at room temperature for $5 \mathrm{~min}$ and then mixed with $20 \mu \mathrm{l}$ of chemiluminescent enhancer containing $1.25 \mathrm{~mm}$ CSPD as a substrate. After incubation in dark for $30 \mathrm{~min}$, the samples were subjected to assays using a luminometer (Gene Light 55; Microtech Nition, Funabashi, Chiba, Japan).

Formazan Assay The number of viable cells was assessed by a formazan assay using Cell Counting Kit-8 (Dojindo Laboratory, Kumamoto, Japan), as described previously. ${ }^{1)}$ In brief, after collecting culture media for chemiluminescent assays, cells in 96-well plates were incubated at $37^{\circ} \mathrm{C}$ for $1.5 \mathrm{~h}$ in medium containing $10 \%$ Cell Counting Kit- 8 assay solution. Absorbance $(450 \mathrm{~nm})$ of formazan generated from WST-8 was measured by Spectea Max 340 (Nihon Molecular Devices, Tokyo, Japan).

Northern Blot Analysis Total RNA was extracted by a single-step method, ${ }^{15)}$ and Northern blot analysis was performed as described before. ${ }^{14)}$ As probes, a HindIII-XbaI SEAP cDNA from pSEAP2-control (BD Biosciences) and a HindIII-EcoRI CYP1A1 cDNA from pCR1000/CYP1A1 ${ }^{16}$ were radiolabeled and used for hybridization. The expression of glyceraldehyde-3-phosphate dehydrogenase was used as a loading control. Densitometric analysis was performed using Scion Image Software (Scion Corporation, Frederick, MD, U.S.A.).

Statistical Analysis Assays were performed in quadruplicate. Data were expressed as means \pm S.E. Statistical analysis was performed using the non-parametric MannWhitney $U$ test to compare data in different groups. $p$ value $<0.05$ was considered to indicate a statistically significant difference.

\section{RESULTS}

Twenty herbal medicine extracts provided by Tsumura \& Co. were initially subjected to rough screening for AhR an- 
tagonistic activity. The samples tested were; TJ-7, TJ-9, TJ10, TJ-12, TJ-18, TJ-23, TJ-36, TJ-41, TJ-43, TJ-47, TJ-48, TJ-53, TJ-54, TJ-62, TJ-83, TJ-87, TJ-100, TJ-107, TJ-108 and TJ-114. The reporter cells HeXS34 cultured in 96-well plates were pretreated with individual extracts at concentrations from 5 to $500 \mu \mathrm{g} / \mathrm{ml}$ for $30 \mathrm{~min}$ and subsequently stimulated with or without 2,3,7,8-TCDD (25 pM) for $24 \mathrm{~h}$. After the treatments, culture media were subjected to chemiluminescent assay to evaluate SEAP activity. Culture media from wells in which cell damage was observed were not used for evaluation. At the concentration of $500 \mu \mathrm{g} / \mathrm{ml}$, more than half of the test samples caused cellular damage. After this initial screening, we selected 4 possible candidates that may have modest antagonistic effects on AhR. Those were; TJ-9 (Formula bupleuri minor; Sho-saiko-to, $50 \mu \mathrm{g} / \mathrm{ml}$ ), TJ-18 (Formula cinnamomi cum aconito et atractylode; Keishi-ka-jutsubu-to, $500 \mu \mathrm{g} / \mathrm{ml}$ ), TJ-23 (Pulvis paeoniae et angelicae; Toki-shakuyaku-san, $500 \mu \mathrm{g} / \mathrm{ml}$ ) and TJ-107 (Pilulae renales plantaginis et achyranthis; Gosha-jinki-gan, $500 \mu \mathrm{g} / \mathrm{ml}$ ). Using these materials, we further tested their antagonistic activity for AhR in more detail at concentrations of 100 and $300 \mu \mathrm{g} / \mathrm{ml}$. Under the unstimulated condition, the level of SEAP in culture medium of HeXS34 was approximately $100-200$ relative light unit (RLU). This level was markedly upregulated to $20000-30000$ RLU in response to $2,3,7,8$ TCDD. When the cells were pretreated with the extracts of TJ-9, TJ-18, TJ-23 and TJ-107, only TJ-9 significantly inhibited activation of AhR in a dose-dependent manner (Fig. 1A). Of note, TJ-9 per se slightly increased the activity of AhR in the absence of 2,3,7,8-TCDD. During this study, cellular damage was not observed in any of extracts tested. This result was further confirmed at lower concentrations of TJ-9. As shown in Fig. 1B, slight, but significant decrease in 2,3,7,8-TCDD-induced AhR activation was observed at concentrations of $10-50 \mu \mathrm{g} / \mathrm{ml}$. At $100 \mu \mathrm{g} / \mathrm{ml}$, the activation of AhR by $2,3,7,8$-TCDD was substantially reduced to $c a$. $50 \%$. Consistent with the result of Fig. 1A, TJ-9 per se induced weak activation of AhR, suggesting that TJ-9 may contain both agonistic and antagonistic constituents.

TJ-9 is composed of 7 raw herb extracts; Bupleuri Radix (Saiko), Pinelliae Tuber (Hange), Scutellariae Radix (Ogon),
Zizyphi Fructus (Taiso), Ginseng Radix (Ninjin), Glycyrrhizae Radix (Kanzo) and Zingiberis Rhizoma (Shokyo). As the second step of investigation, we tested effects of individual herb extracts on the activation of AhR by 2,3,7,8TCDD. HeXS34 cells were pretreated with individual samples at $250 \mu \mathrm{g} / \mathrm{ml}$ for $30 \mathrm{~min}$ and subsequently stimulated with or without $2,3,7,8$-TCDD for $24 \mathrm{~h}$. SEAP assay revealed that Glycyrrhizae Radix and Scutellariae Radix were responsible for the antagonistic effect of TJ-9 on AhR (Fig. 2A). These herb extracts dramatically inhibited the induction of SEAP by 2,3,7,8-TCDD, and little agonistic activity for AhR was observed. Interestingly, some components including $\mathrm{Bu}$ pleuri Radix (the major component of TJ-9) and Zingiberis Rhizoma per se significantly $(p<0.05)$ activated AhR and rather enhanced the activation of AhR by 2,3,7,8-TCDD. Pinelliae Tuber and Ginseng Radix also modestly enhanced 2,3,7,8-TCDD-triggered AhR activation. These results suggested that, although TJ-9 has the potential for suppression of AhR activation triggered by dioxin, it contains both agonists and antagonists of AhR.

We further examined dose-dependent effects of Glycyrrhizae Radix and Scutellariae Radix on the activation of AhR by 2,3,7,8-TCDD at lower concentrations. Treatment with $50 \mu \mathrm{g} / \mathrm{ml}$ Scutellariae Radix significantly inhibited TCDD-induced AhR activation (Fig. 2B). Scutellariae Radix at lower concentrations $(1-10 \mu \mathrm{g} / \mathrm{ml})$ was ineffective in suppression of AhR activation by 2,3,7,8-TCDD. At concentrations higher than $100 \mu \mathrm{g} / \mathrm{ml}$, Scutellariae Radix induced cellular damage in HeXS34 cells (data not shown). Similarly, treatment with Glycyrrhizae Radix significantly inhibited TCDD-induced activation of AhR at concentrations of 100 and $150 \mu \mathrm{g} / \mathrm{ml}$, and the herb extract at lower concentrations was ineffective (Fig. 2C). At concentrations higher than $250 \mu \mathrm{g} / \mathrm{ml}$, Glycyrrhizae Radix induced cellular damage (data not shown). Both raw herb extracts at high concentrations paradoxically caused modest activation of AhR. It is possibly because both extracts contain some AhR agonists as well as antagonists, as shown later.

Ohtake et al. previously reported high-performance liquid chromatography-based analysis of pharmacologically active ingredients in the individual raw herb extracts contained in
(A)

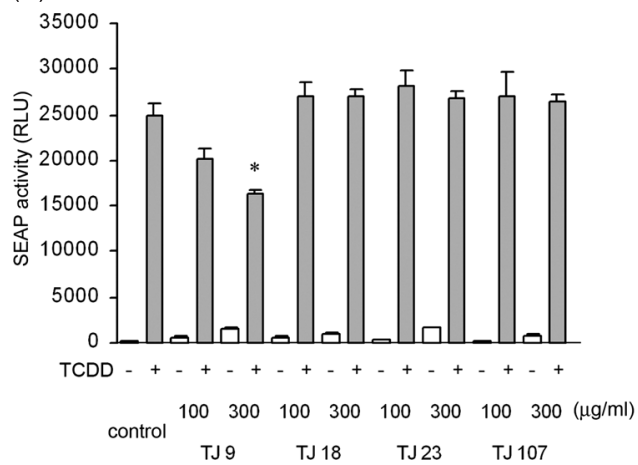

(B)

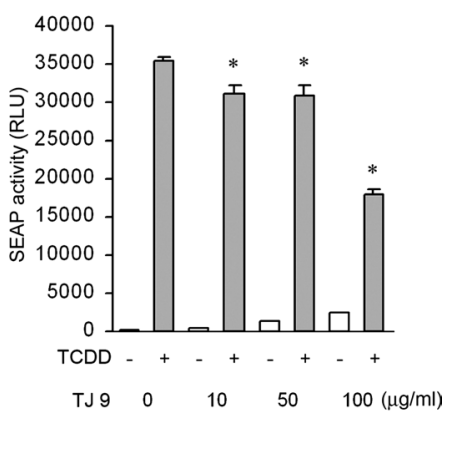

Fig. 1. Screening of the Potential of Herbal Medicines for Inhibition of Aryl Hydrocarbon Receptor (AhR) Activation Triggered by Dioxin

(A) Four herbal medicines that may have antagonistic effects on AhR were selected by initial rough screening and re-tested at concentrations of 100 and $300 \mu \mathrm{g} / \mathrm{ml}$. The materials tested were TJ-9 (Formula bupleuri minor; Sho-saiko-to), TJ-18 (Formula cinnamomi cum aconito et atractylode; Keishi-ka-jutsu-bu-to), TJ-23 (Pulvis paeoniae et angelicae; Toki-shakuyaku-san) and TJ-107 (Pilulae renales plantaginis et achyranthis; Gosha-jinki-gan). HeXS34 reporter cells were pretreated with these agents individually and exposed to 25 p 2,3,7,8-tetrachlorodibenzo- $p$-dioxin $(2,3,7,8$-TCDD) for $24 \mathrm{~h}$. Activity of secreted alkaline phosphatase (SEAP) in culture media was evaluated by chemiluminescent assay. (B) Dose-dependent effect of TJ-9 on the activation of AhR by 2,3,7,8-TCDD. Assays were performed in quadruplicate. Data are presented as means \pm S.E., and asterisks indicate statistically significant differences $(p<0.05)$. 
(A)

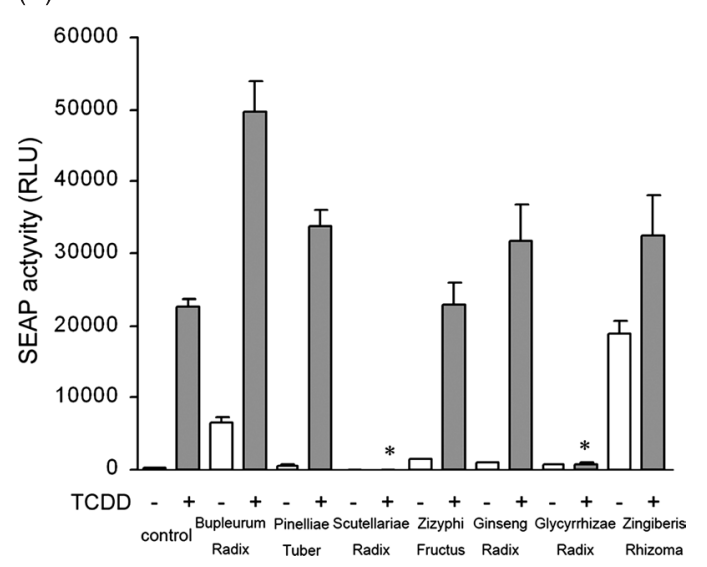

(B)

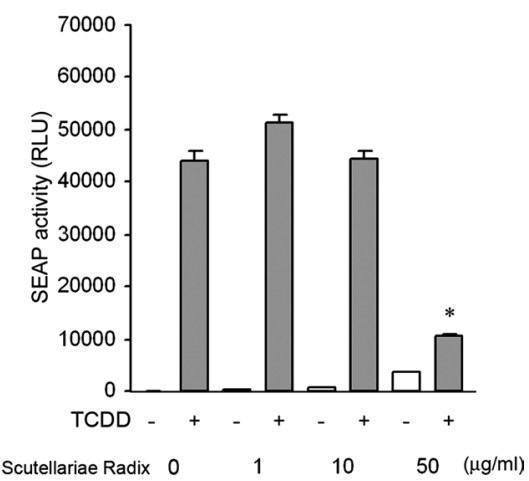

(C)

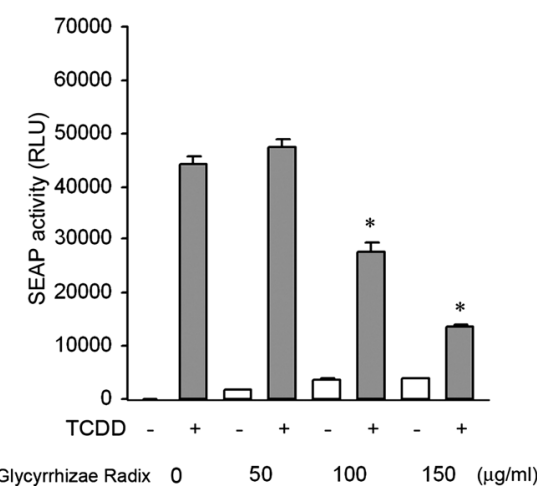

Fig. 2. Potential of Individual Raw Herb Extracts in TJ-9 for Inhibition of Dioxin-Induced AhR Activation

(A) HeXS34 cells were pretreated with Bupleuri Radix, Pinelliae Tuber, Scutellariae Radix, Zizyphi Fructus, Ginseng Radix, Glycyrrhizae Radix or Zingiberis Rhizoma at $250 \mu \mathrm{g} / \mathrm{ml}$ for $30 \mathrm{~min}$ and subsequently stimulated with $2,3,7,8$-TCDD for $24 \mathrm{~h}$. Activity of SEAP was evaluated by chemiluminescent assay. (B, C) Dose-dependent inhibitory effects of Scutellariae Radix (B) and Glycyrrhizae Radix (C) on the activation of AhR by 2,3,7,8-TCDD.

TJ-9. ${ }^{17)}$ In Glycyrrhizae Radix, they identified 13 low molecular mass constituents including liquiritin, liquiritin apioside, liquiritigenin, isoliquiritin, isoliquiritin apioside, isoliquiritigenin, glycyrrhizin, glycyrrhizic acid, glycycoumarin, genistein, licopyranocoumarin, licocoumarone and glycyrrhisoflavone. Among these constituents, we tested 7 available components including genistein, licopyranocoumarin, glycyrrhizic acid, liquiritin, liquiritin apioside, isoliquiritin and glycycoumarin at concentrations that do not cause cellular damage. As shown in Fig. 3A, substantial suppression of dioxin-induced $\mathrm{AhR}$ activation was observed in genistein and licopyranocoumarin at concentrations lower than $10 \mu \mathrm{g} / \mathrm{ml}$. At relatively higher concentrations $(50-150 \mu \mathrm{g} / \mathrm{ml})$, glycyrrhizic acid also showed a weak antagonistic effect. Significant suppression of AhR was not observed in liquiritin, liquiritin apioside and isoliquiritin at concentrations of $<150 \mu \mathrm{g} / \mathrm{ml}$. In the case of glycycoumarin, we used only low concentrations because at concentrations higher than $5 \mu \mathrm{g} / \mathrm{ml}$, this compound induced cellular damage (data not shown). Of note, substantial agonistic activity for AhR was not detected in any of these agents at any concentrations tested.

The SEAP activity may be influenced by alteration in the cell number caused by cell proliferation or cell death. To confirm our conclusion described above, the number of viable cells was evaluated using formazan assay after sampling of culture media for SEAP assay. The SEAP values were normalized by the level of formazan, and effects of genistein, licopyranocoumarin and glycyrrhizic acid were re-evaluated. As shown in Fig. 3B, dramatic suppression of AhR activation was observed by the treatment with genistein and licopyranocoumarin. The suppressive effect of glycyrrhizic acid was only modest. These results suggested that the major constituents responsible for suppression of AhR by Glycyrrhizae Radix were genistein and licopyranocoumarin.

In Scutellariae Radix, Ohtake et al. identified 7 low molecular mass constituents including baicalin, baicalein, wogonin-7-O-glucuronoside, wogonin, skullcapflavone II, daidzein and arginine. ${ }^{17)}$ Among these constituents, we tested 5 available components including baicalein, baicalin, wogonin, skullcapflavone II and daidzein at concentrations that do not cause cellular damage. As shown in Fig. 4A, substantial suppression of dioxin-induced AhR activation was observed in baicalein, baicalin and wogonin at low concentrations less than $10 \mu \mathrm{g} / \mathrm{ml}$. At relatively higher concentrations (15$25 \mu \mathrm{g} / \mathrm{ml}$ ), daidzein also showed a weak antagonistic effect. In contrast, significant suppression of AhR was not observed in skullcapflavone II within the range of non-toxic dosages. At concentrations higher than $5 \mu \mathrm{g} / \mathrm{ml}$, skullcapflavone II induced cellular damage (data not shown). Of note, substantial, dose-dependent agonistic activity for AhR was observed exclusively in baicalin, suggesting that it is a partial agonist with modest antagonistic activity.

To confirm our conclusion, we evaluated the number of viable cells using formazan assay after sampling of culture media. The SEAP values were normalized by the level of formazan, and effects of baicalein, baicalin, wogonin and daidzein were re-evaluated. As shown in Fig. 4B, significant suppression of $\mathrm{AhR}$ was observed by the treatment with these agents, confirming that all of these constituents were responsible for suppression of AhR by Scutellariae Radix.

$\alpha \mathrm{NF}$ is a well-known, popularly used antagonist of AhR. To evaluate the antagonistic effectiveness of herbal constituents quantitatively, we compared their potential with that of $\alpha \mathrm{NF}$. HeXS34 cells were pretreated with $\alpha \mathrm{NF}$ and herbal components at various non-toxic concentrations, exposed to $2,3,7,8$-TCDD and subjected to chemiluminescent assay to evaluate SEAP activity. The results showed that, compared with $\alpha \mathrm{NF}$, the antagonistic potential of herbal constituents was relatively weaker (Fig. 5). That is, substantial suppression $(50 \%)$ of SEAP activity by $\alpha \mathrm{NF}$ was observed at approximately $0.1 \mu \mathrm{M}$, whereas herbal antagonists required 
(A)
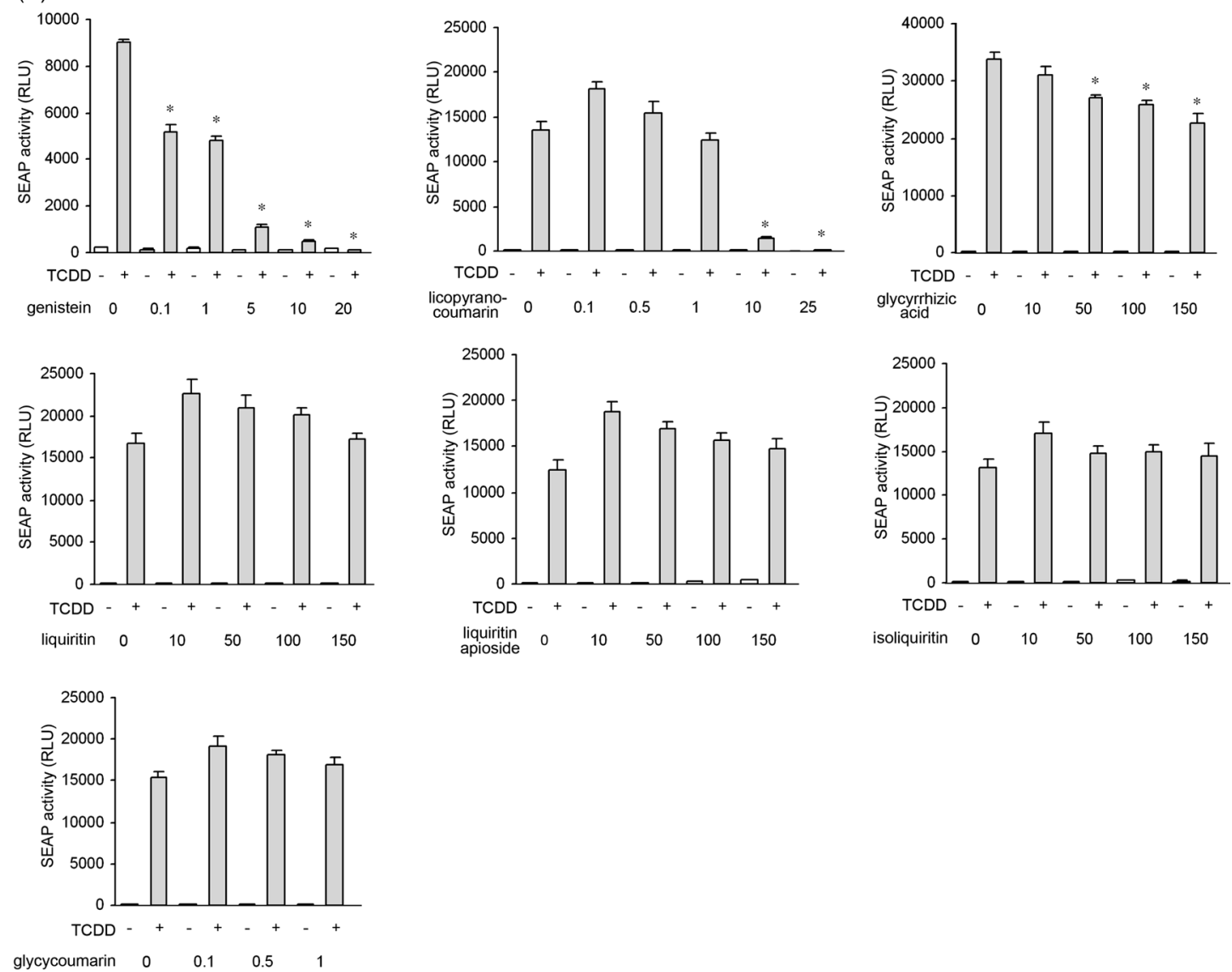

(B)
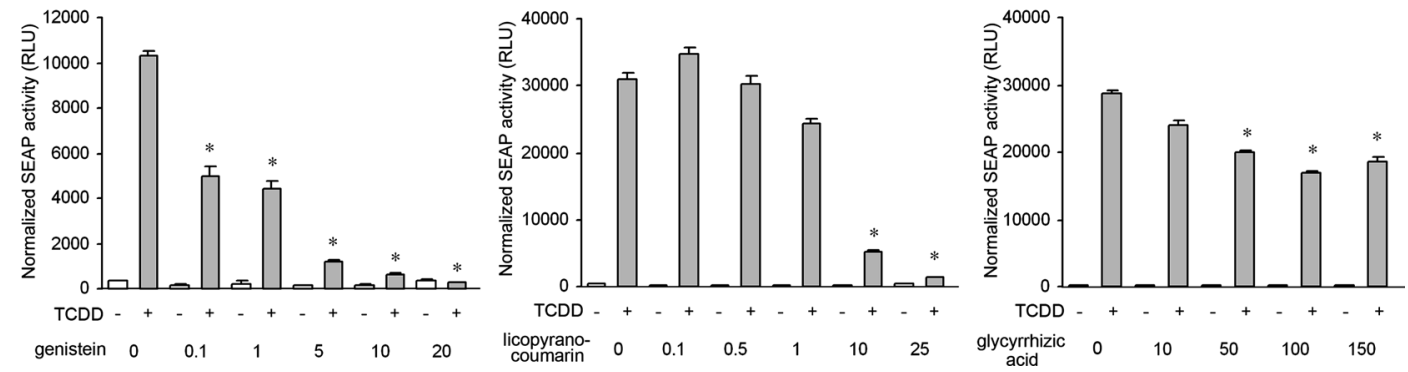

Fig. 3. Identification of AhR Antagonists Contained in Glycyrrhizae Radix

(A) HeXS34 cells were treated with constituents of Glycyrrhizae Radix including genistein, licopyranocoumarin, glycyrrhizic acid, liquiritin, liquiritin apioside, isoliquiritin or glycycoumarin at non-toxic concentrations and exposed to 2,3,7,8-TCDD for $24 \mathrm{~h}$. Activity of SEAP was evaluated by chemiluminescent assay. (B) After sampling of culture media, the cells were subjected to formazan assay to evaluate cell viability. Activity of SEAP was normalized by the number of viable cells, and effects of genistein, licopyranocoumarin and glycyrrhizic acid were re-evaluated.

higher concentrations $(1-100 \mu \mathrm{M})$ to achieve the similar inhibition. Of note, all herbal components per se affected neither activity of SEAP nor the chemiluminescent assay system (data not shown).

In the screening described above, we used SEAP as a reporter molecule to evaluate the activity of AhR. However, we recently found that activity of SEAP produced by reporter cells is down-regulated by endoplasmic reticulum (ER) stress independently of transcriptional regulation. ${ }^{18)}$ This phenomenon was observed in a wide range of cell types triggered by various ER stress inducers. ${ }^{19)}$ To exclude a possibility that the suppression of SEAP by herbal constituents was via induction of ER stress, additional experiments were conducted; i.e., 1) evaluation of SEAP mRNA levels by Northern blot analysis, and 2) assessment of SEAP activity in HeSS8 cells constitutively secreting SEAP after the treatment with herbal components. First, HeXS34 cells were exposed to 2,3,7,8TCDD in the absence or presence of wogonin, daidzein, genistein, licopyranocoumarin, baicalein or baicalin, and expression of SEAP mRNA was evaluated by Northern blot analysis. As shown in Fig. 6A, all of these chemicals except for daidzein substantially suppressed SEAP mRNA induced by dioxin. Of note, consistent with the results shown in Figs. $4 \mathrm{~A}$ and $\mathrm{B}$, modest induction of SEAP was observed only in $25 \mu \mathrm{g} / \mathrm{ml}$ baicalin, but not others. Second, HeSS8 cells were treated with licopyranocoumarin, genistein, baicalein, 
(A)
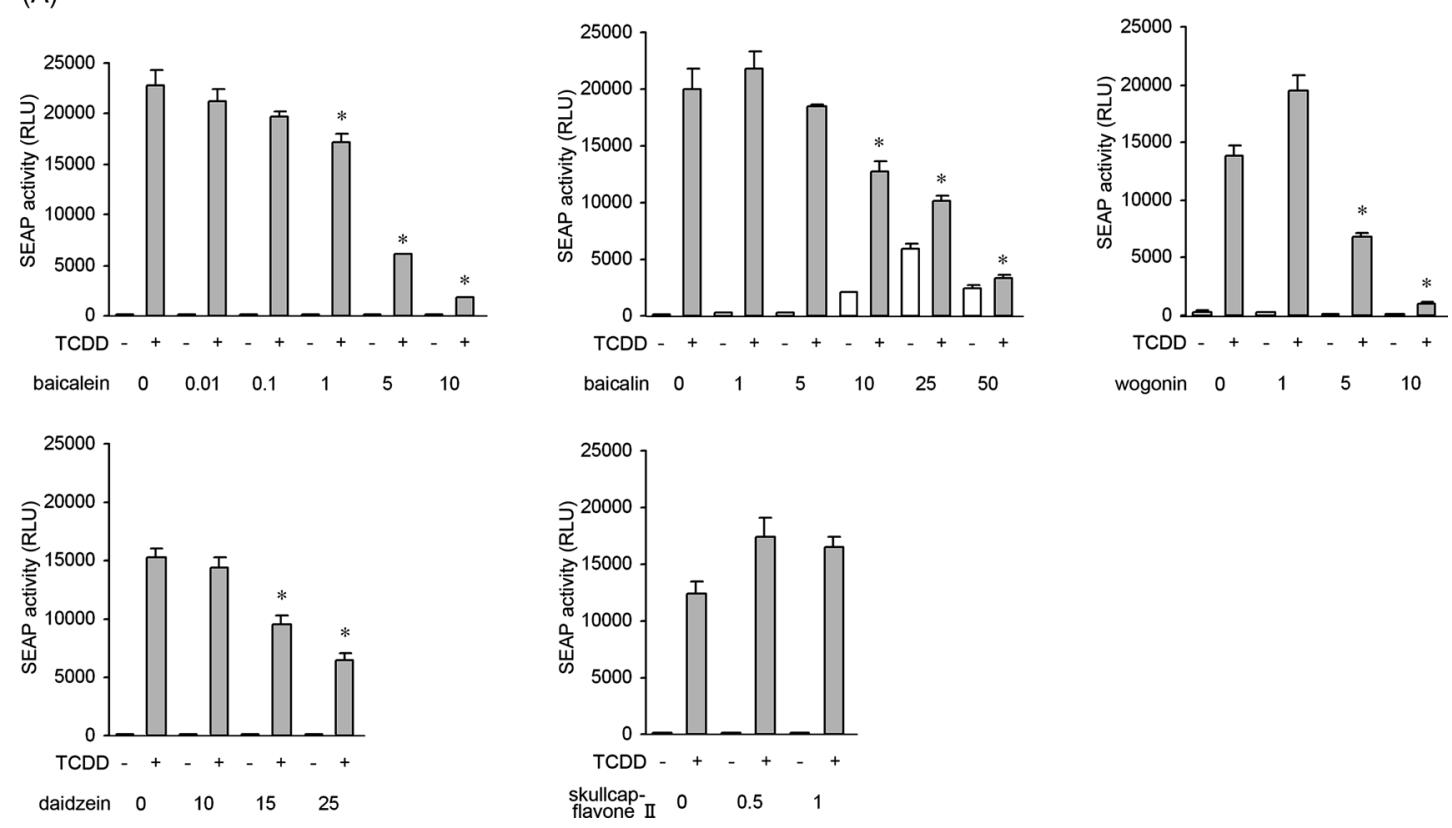

(B)
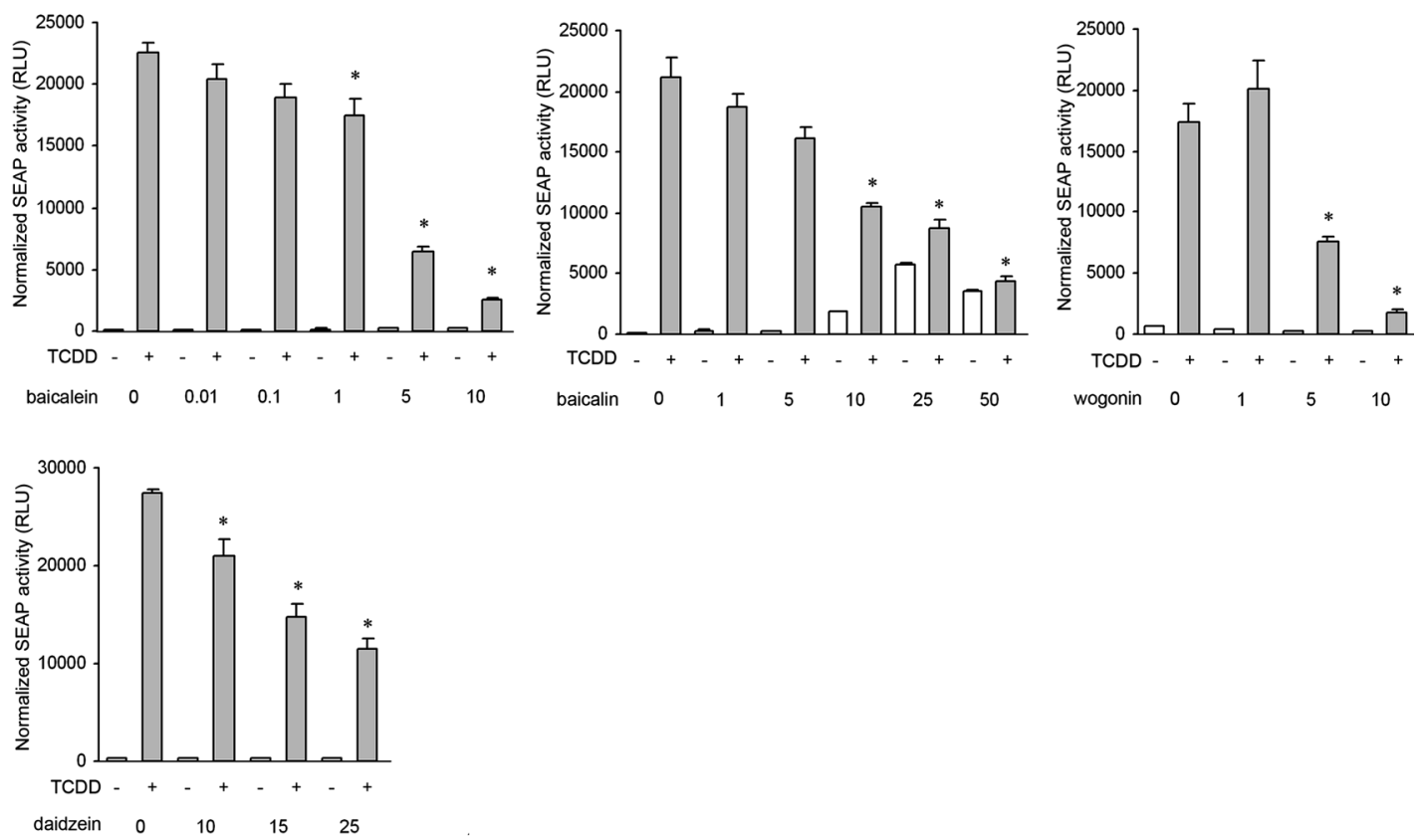

Fig. 4. Identification of AhR Antagonists Contained in Scutellariae Radix

(A) HeXS34 cells were treated with constituents of Scutellariae Radix including baicalein, baicalin, wogonin, daidzein or skullcapflavone II at non-toxic concentrations and exposed to 2,3,7,8-TCDD for $24 \mathrm{~h}$. Activity of SEAP was evaluated by chemiluminescent assay. (B) After sampling of culture media, the cells were subjected to formazan assay to evaluate cell viability. Activity of SEAP was normalized by the number of viable cells, and effects of baicalein, baicalin, wogonin and daidzein were re-evaluated.

baicalin, wogonin and daidzein at effective concentrations, and the culture media and cells were subjected to SEAP assay and formazan assay, respectively. The SEAP values were then normalized by the levels of formazan generated and used for evaluation. The results showed that the SEAP levels produced by HeSS 8 cells were not affected by baicalein, baicalin or licopyranocoumarin, and slight repression of the SEAP levels was observed in genistein, wogonin and daidzein (data not shown). Taken together, these results confirmed that the suppression of SEAP activity by herbal constituents was not caused by ER stress.
To further confirm our conclusion, we examined effects of individual herbal constituents on 2,3,7,8-TCDD-induced expression of CYP1A1, an endogenous marker of AhR activation. For this purpose, we used human hepatoma HepG2 cells to examine whether the antagonistic effects of herbal components are observed not only in a particular murine cell line but also in other cell types, especially in human cells. HepG2 cells were treated with individual herbal components, exposed to 2,3,7,8-TCDD and subjected to Northern blot analysis of CYP1A1. As shown in Fig. 6B, all substances tested, including baicalein, baicalin, genistein, wogonin, daidzein 

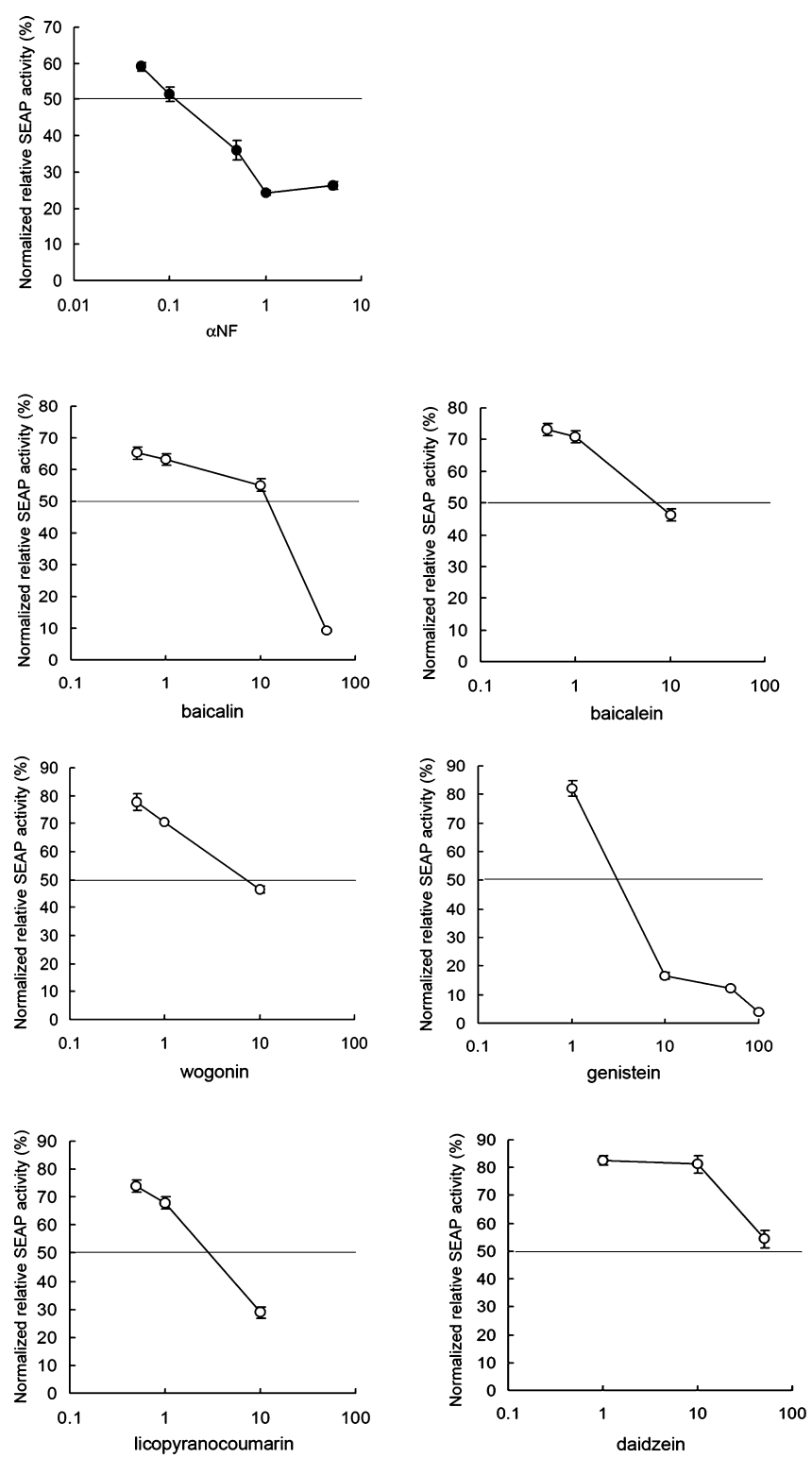

Fig. 5. Comparison of the Antagonistic Potential of Herbal Constituents with That of $\alpha$-Naphthoflavone ( $\alpha \mathrm{NF}$ )

HeXS34 cells were pretreated with $0.05-5 \mu \mathrm{M} \alpha \mathrm{NF}$ and herbal components (baicalein, baicalin, genistein, wogonin, daidzein licopyranocoumarin, glycyrrhizic acid) at non-toxic concentrations $(1-100 \mu \mathrm{M})$, exposed to $2,3,7,8$-TCDD for $24 \mathrm{~h}$ and subjected to chemiluminescent assay to evaluate SEAP activity.

and licopyranocoumarin, inhibited induction of CYP1A1. Of note, baicalein, genistein, wogonin and daidzein also suppressed basal expression of CYP1A1.

We recently reported that cigarette smoke contains high levels of agonists for AhR. Cigarette smoke causes a variety of pathologies, some of which are also caused by dioxins and dioxin-like chemicals. We tested effects of AhR antagonists identified in herbal medicines on the activation of AhR by cigarette smoke. CSE was prepared as described in Materials and Methods. HeXS34 cells were pretreated with baicalein, baicalin, genistein, wogonin, daidzein or licopyranocoumarin and stimulated with CSE (1\%). After incubation for $24 \mathrm{~h}$, the media and cells were subjected to chemiluminescent assay and formazan assay, respectively. The SEAP values were normalized by the level of formazan, and effects of individual antagonists were evaluated. Although baicalin, genistein,
(A)
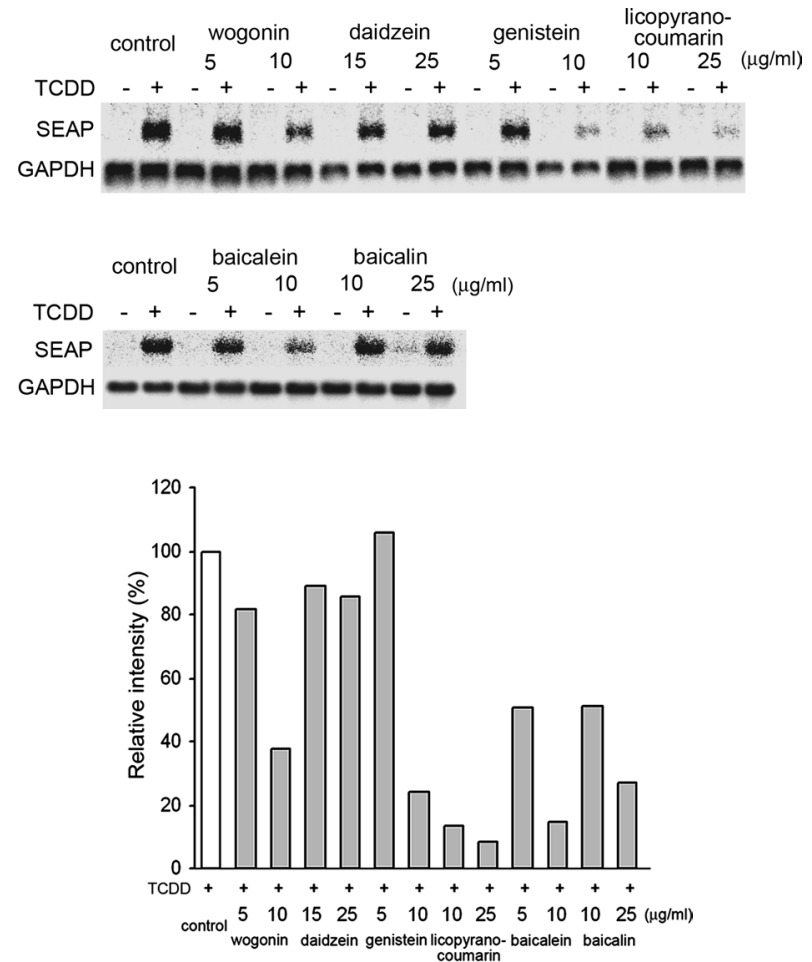

(B)
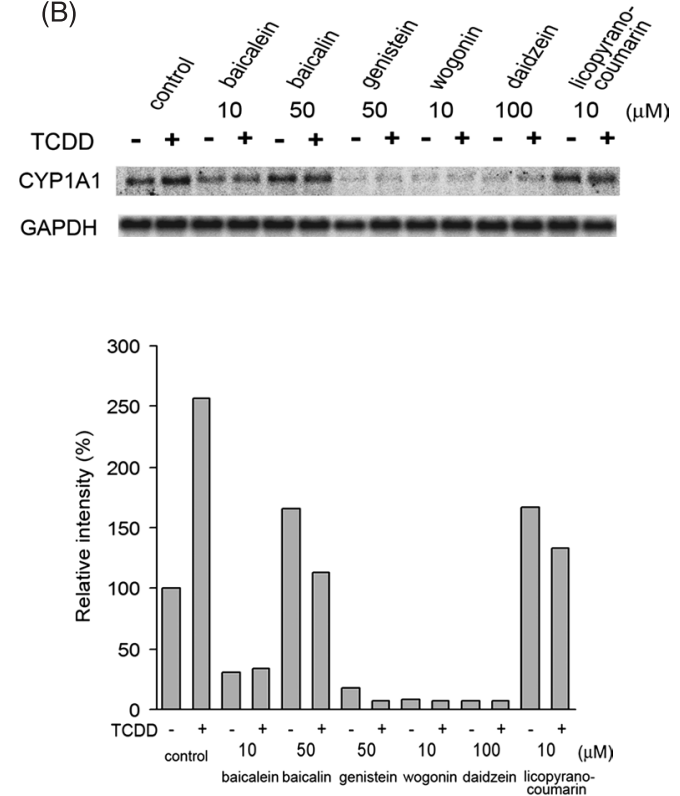

Fig. 6. Northern Blot Analyses of Exogenous and Endogenous Indicators for AhR Activation in Dioxin-Exposed Cells Pretreated with Herbal AhR Antagonists

(A) HeXS34 cells were exposed to 2,3,7,8-TCDD in the absence or presence of wogonin $(5,10 \mu \mathrm{g} / \mathrm{ml})$, daidzein $(15,25 \mu \mathrm{g} / \mathrm{ml})$, genistein $(5,10 \mu \mathrm{g} / \mathrm{ml})$, licopyranocoumarin $(10,25 \mu \mathrm{g} / \mathrm{ml})$, baicalein $(5,10 \mu \mathrm{g} / \mathrm{ml})$ and baicalin $(10,25 \mu \mathrm{g} / \mathrm{ml})$ for $4 \mathrm{~h}$, and expression of SEAP mRNA was evaluated by Northern blot analysis. (B) HepG2 cells were exposed to $2,3,7,8-\mathrm{TCDD}$ in the absence or presence of baicalein $(10 \mu \mathrm{M})$, baicalin $(50 \mu \mathrm{M})$, genistein $(50 \mu \mathrm{M})$, wogonin $(10 \mu \mathrm{M})$, daidzein $(100 \mu \mathrm{M})$ and licopyranocoumarin $(100 \mu \mathrm{M})$ for $4 \mathrm{~h}$, and expression of cytochrome P450 1A1 (CYP1A1) mRNA was evaluated by Northern blot analysis. As a loading control, expression of glyceraldehyde-3-phosphate dehydrogenase (GAPDH) is shown. Intensity of individual signals for SEAP and CYP1A1 was normalized by the intensity of GAPDH, and the results are shown as graphs. 


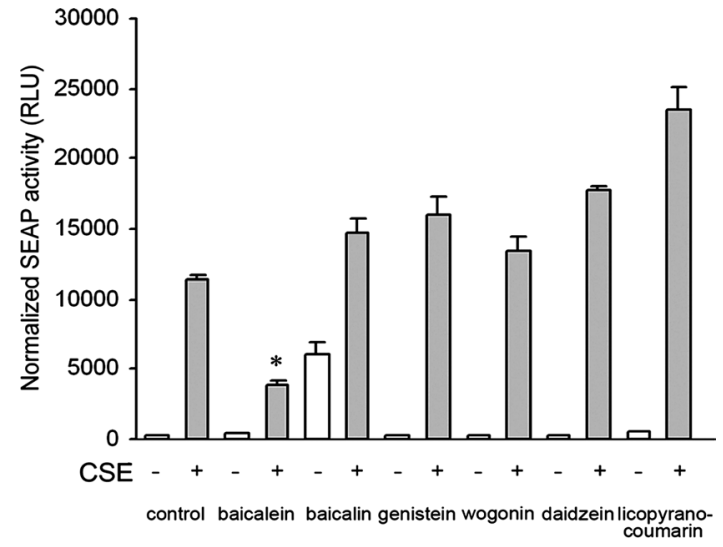

Fig. 7. Potential of Individual AhR Antagonists for Inhibition of Cigarette Smoke-Induced AhR Activation

HeXS34 cells were pretreated with baicalein $(10 \mu \mathrm{g} / \mathrm{ml})$, baicalin $(25 \mu \mathrm{g} / \mathrm{ml})$, genistein $(10 \mu \mathrm{g} / \mathrm{ml})$, wogonin $(10 \mu \mathrm{g} / \mathrm{ml})$, daidzein $(25 \mu \mathrm{g} / \mathrm{ml})$ or licopyranocoumarin $(25 \mu \mathrm{g} / \mathrm{ml})$ for $30 \mathrm{~min}$ and exposed to cigarette smoke extracts (CSE; $1 \%)$. After incubation for $24 \mathrm{~h}$, the media and cells were subjected to chemiluminescent assay and formazan assay, respectively. The SEAP values were normalized by the level of formazan, and effects of individual antagonists were evaluated.

wogonin, daidzein and licopyranocoumarin effectively inhibited dioxin-induced activation of AhR, they did not suppress activation of AhR by CSE (Fig. 7). Only baicalein significantly inhibited the effect of CSE. These results suggested that, among several AhR antagonists in herbal extracts, baicalein uniquely had the potential for attenuation of cigarette smoke-triggered $\mathrm{AhR}$ activation.

\section{DISCUSSION}

Aberrant activation of AhR by xenobiotic ligands results in various pathologies. For the purpose of prevention and treatment of the diseases related to activation of the AhR-XRE/DRE pathway, development of AhR antagonists will be required. Towards this end, we aimed in the present study at hunting for potent AhR antagonists in herbal medicines using the DRESSA bioassay. Our data elucidated that Formula bupleuri minor (TJ-9) and its raw herb components Glycyrrhizae Radix and Scutellariae Radix have the potential for suppression of dioxin-induced AhR activation. Interestingly, some components of Formula bupleuri minor including Bupleuri Radix, a major component of Formula bupleuri minor, and Zingiberis Rhizoma significantly activated AhR and rather enhanced dioxin-induced activation of the AhR-XRE/DRE pathway. Other components Pinelliae Tuber and Ginseng Radix also modestly enhanced dioxin-triggered activation of AhR. Even in Glycyrrhizae Radix and Scutellariae Radix, weak agonistic activity for AhR was observed. These results suggested that, although Formula bupleuri minor has the potential for suppression of dioxin-triggered activation of $\mathrm{AhR}$, it consists of both agonistic and antagonistic components. To our knowledge, this is the first to demonstrate influences of herbal medicines and raw herb extracts on the activity of the AhR-XRE/DRE pathway.

In this report, we identified that Formula bupleuri minor significantly inhibited dioxin-triggered activation of AhR. Formula bupleuri minor is one of the most popular herbal medicines and has been used for the treatment of inflammatory diseases including hepatitis, bronchitis/pneumonia and enterogastritis. In particular, long-term administration with Formula bupleuri minor has been used for the treatment of chronic hepatitis to improve liver function as well as for prevention of hepatocellular carcinoma. ${ }^{20,21)}$ However, to date, little has been elucidated about mechanisms underlying the therapeutic effects of Formula bupleuri minor. It is known that the $5^{\prime}$-regulatory regions of a number of inflammationassociated genes including inducible nitric oxide synthase, cyclooxygenase-2, lipoxygenase, p50/p65 NF- $\kappa \mathrm{B}$ subunits, RANTES, IL- $\beta$, IL-2 receptors, IL-8 receptors, tumor necrosis factor receptor $\mathrm{p} 75 / \mathrm{p} 80$ and intercellular adhesion molecule contain XRE. ${ }^{9)}$ The anti-inflammatory effects of Formula bupleuri minor could be due to inhibition of elaborating these proinflammatory molecules via suppression of the AhR-XRE/DRE pathway. A previous report also showed that activation of AhR plays crucial roles in the promotion of carcinogenesis. ${ }^{22}$ For example, transgenic mice expressing constitutively active AhR are much more susceptible to hepatocarcinogenesis triggered by $N$-nitrosodiethylamine. ${ }^{23)}$ Formula bupleuri minor could be useful for the treatment of some tumors caused by aberrant activation of AhR.

An interesting finding in the present report is that, although Formula bupleuri minor inhibits activation of AhR by 2,3,7,8-TCDD, it consists of both agonistic and antagonistic constituents for AhR. We found that Glycyrrhizae Radix and Scutellariae Radix substantially inhibited activation of AhR by 2,3,7,8-TCDD, and little agonistic activity for AhR was observed. However, Bupleuri Radix and Zingiberis Rhizoma significantly activated AhR and rather enhanced activation of AhR by 2,3,7,8-TCDD. Pinelliae Tuber and Ginseng Radix also modestly reinforced dioxin-triggered activation of AhR. It might, therefore, be feasible to prepare more effective, anti-inflammatory formula of herbal medicine by, for example, combination of Glycyrrhizae Radix and Scutellariae Radix without other components.

Previous reports showed that some substances identified in the present study may have the potential for activation of AhR. For example, in contrast to our current results, two groups reported baicalein, baicalin, genistein and daidzein to be agonists of AhR using a reporter assay and a receptor binding assay. ${ }^{24,25)}$ Using a gel mobility shift assay, Ashida showed that baicalein, but not genistein and daidzein, was a weak agonist of AhR. ${ }^{26)}$ Using the DRESSA bioassay, we have identified that baicalin, but not other compounds tested including baicalein, genistein and daidzein, was a weak agonist of AhR. The reason for the discrepancy from report to report is currently unclear, but it is possibly due to differences in the quality of test materials and type of assay systems utilized, as suggested by Zhang et al. ${ }^{24)}$ In contrast to the extensive seeking for AhR agonists, information is very limited regarding antagonists of $\mathrm{AhR}$. In the present report, we identified licopyranocoumarin, glycyrrhizic acid, genistein, baicalein, wogonin and daidzein are antagonists of AhR. These findings are, in part, consistent with some previous reports. For example, using a gel mobility shift assay, Ashida reported that baicalein was an antagonist of $\mathrm{AhR} .^{26)}$ Using an ELISA-based binding assay, Amakura et al. also showed that baicalein and genistein were AhR antagonists. ${ }^{27)}$

Currently, the details for the "antagonistic" potential of these chemicals are unclear. We cannot exclude a possibility that these substances act as inhibitors of AhR not via the 
AhR antagonism but through other indirect mechanisms such as alteration of AhR affinity or inhibition of relevant kinases. Further investigation will be required to clarify this issue.

Recent investigation suggested that natural products such as fruits and vegetables contain active entities that affect activity of AhR. The best example is resveratrol (3,5,4'-trihydroxystilbene), a natural polyphenolic phytoalexin abundantly contained in grapes and red wine. Using reporter assays, previous reports showed that resveratrol was antagonistic against dioxin-triggered activation of AhR. ${ }^{28)}$ Vegetable constituents also have the potential for modulating AhR activity $^{24-27)}$; some may act as AhR agonists and others function as antagonists. In many cases, antagonistic constituents suppress dioxin-induced AhR activation, whereas, when used alone, they also exhibit modest agonistic activity. ${ }^{12)}$ To seek for pure AhR antagonists, we initiated the present investigation using traditional herbal medicines as initial sources. It is because: 1) Herbal medicines, composed of several different raw herb extracts, have been used for the treatment of a variety of diseases in Eastern countries over 1500 years. It is reasonable to speculate that biologically active substances have been enriched in herbal medicines through the long-term clinical experiences. 2) Herbal medicines have also been used in the modern medicine, and its clinical safety in humans has already been confirmed. It means that if some herbal medicines possess strong potential as AhR antagonists, it is feasible to use them in clinics without through testing stages. 3) In Japan, a number of herbal medicines are manufactured on a modern industrial scale in which the quality and quantity of ingredients are standardized under strict, scientific controls. 4) The chemical constituents of individual raw herb extracts have been investigated in detail by pharmaceutical industries. The information should provide us with great advantages for identifying inhibitory substances for AhR. Indeed, in the present study, we successfully found several antagonists of AhR using herbal medicine extracts as initial materials and the DRESSA bioassay as the screening system.

Activation of $\mathrm{AhR}$ mediates toxicities of dioxins and dioxin-like substances. Based on this current concept, AhR antagonists identified here might be useful for prevention and treatment of pathologies associated with aberrant activation of AhR, including cigarette smoke-related diseases. However, unexpectedly, although baicalein, baicalin, genistein, wogonin, daidzein and licopyranocoumarin effectively inhibited dioxin-induced activation of $\mathrm{AhR}$, only baicalein, but not others, significantly inhibited the CSE-induced activation of the dioxin pathway. Currently, the reason for this result is unclear, but our finding raises a possibility that baicalein might be a unique AhR antagonist that blocks activation of the AhR-XRE/DRE pathway triggered by cigarette smoke and could attenuate progression of some cigarette smoke-related disorders.

Currently, there are no data on the quantity of baicalein in Formula bupleuri minor and Scutellariae Radix. In the present study, we used 5-10 $\mu \mathrm{g} / \mathrm{ml}$ baicalein to demonstrate effective inhibition of AhR activation by 2,3,7,8-TCDD and CSE. Formula bupleuri minor inhibited activation of AhR at $10-100 \mu \mathrm{g} / \mathrm{ml}$, and the content of Scutellariae Radix in Formula bupleuri minor is $12.5 \%$. The concentrations of baicalein we used, therefore, may be too high to prove the important contribution of this substance to the inhibitory effects of Formula bupleuri minor and Scutellariae Radix on AhR. Additional investigation may be required to further confirm our conclusion.

Acknowledgments We thank Tsumura \& Co. for providing herbal medicine extracts, raw herb extracts and their constituents. This work was supported by grants from The Kampo Science Organization and The Smoking Research Foundation, and in part, from Grants-in-Aid for Scientific Research from the Ministry of Education, Culture, Sports, Science and Technology, Japan (No. 16390243 and No. 17651026 to M. K.). Ayumi Kasai is a Research Fellow of Japan Society of the Promotion Science.

\section{REFERENCES}

1) Kasai A., Hiramatsu N., Hayakawa K., Yao J., Maeda S., Kitamura M., Cancer Res., 66, 7143-7150 (2006).

2) Birnbaum L. S., Tuomisto J., Food Addit. Contam., 17, 275-288 (2000).

3) Tohyama C., Environ. Sci., 9, 37-50 (2003).

4) Mimura J., Fujii-Kuriyama Y., Biochim. Biophys. Acta, 1619, 263268 (2003).

5) Denison M. S., Pandini A., Nagy S. R., Baldwin E. P., Chem. Biol. Interact., 141, 3-24 (2002).

6) Fernandez-Salguero P. M., Hilbert D. M., Rudikoff S., Ward J. M., Gonzalez F. J., Toxicol. Appl. Pharmacol., 140, 173-179 (1996).

7) Andersson P., McGuire J., Rubio C., Gradin K., Whitelaw M. L., Pettersson S., Hanberg A., Poellinger L., Proc. Natl. Acad. Sci. U.S.A., 99, 9990_-9995 (2002).

8) Andersson P., Ridderstad A., McGuire J., Pettersson S., Poellinger L., Hanberg A., Biochem. Biophys. Res. Commun., 302, 336-341 (2003).

9) Savouret J. F., Berdeaux A., Casper R. F., Nutr. Metab. Cardiovasc. Dis., 13, 104-113 (2003).

10) Sasco A. J., Secretana M. B., Straif K., Lung Cancer, 45, 53-59 (2004).

11) Kasai A., Hiramatsu N., Meng Y., Yao J., Takeda M., Maeda S., Kitamura M., Anal. Biochem., 335, 73-80 (2004).

12) Kasai A., Hiramatsu N., Meng Y., Yao J., Maeda S., Kitamura M., Anal. Biochem., 337, 84-88 (2005).

13) Kasai A., Yao J., Yamauchi K., Hiramatsu N., Hayakawa K., Meng Y., Maeda S., Kitamura M., Toxicol. Appl. Pharmacol., 211, 11-19 (2006).

14) Hayakawa K., Meng Y., Hiramatsu N., Kasai A., Yamauchi K., Yao J., Kitamura M., J. Immunol., 176, 2529-2537 (2006).

15) Chomczynski P., Sacchi N., Anal. Biochem., 162, 156-159 (1987)

16) Shibazaki M., Takeuchi T., Ahmed S., Kikuchi H., J. Biol. Chem., 279, 3869-3876 (2004)

17) Ohtake N., Nakai Y., Yamamoto M., Sakakibara I., Takeda S., Amagaya S., Aburada M., J. Chromatogr. B: Analyt. Technol. Biomed. Life Sci., 812, 135-148 (2004).

18) Hiramatsu N., Kasai A., Hayakawa K., Nagai K., Kubota T., Yao J., Kitamura M., J. Immunol. Methods, 315, 202-207 (2006).

19) Hiramatsu N., Kasai A., Hayakawa K., Yao J., Kitamura M., Nucleic Acids Res., 34, e93 (2006).

20) Borchers A. T., Sakai S., Henderson G. L., Harkey M. R., Keen C. L., Stern J. S., Terasawa K., Gershwin M. E., J. Ethnopharmacol., 73, 113 (2007)

21) Shimizu I., J. Gastroenterol. Hepatol., SI, D84-D90 (2000).

22) Nebert D. W., Dalton T. P., Okey A. B., Gonzalez F. J., J. Biol. Chem., 279, 23847-23850 (2004)

23) Moennikes O., Loeppen S., Buchmann A., Andersson P., Ittrich C., Poellinger L., Schwarz M., Cancer Res., 64, 4707-4710 (2004).

24) Zhang S., Qin C., Safe S. H., Environ. Health Perspect., 11, $1877-$ 1882 (2003).

25) Amakura Y., Tsutsumi T., Nakamura M., Kitagawa H., Fujino J., Sasaki K., Toyoda M., Yoshida T., Maitani T., Biol. Pharm. Bull., 26, 532-539 (2003).

26) Ashida H., Biofactors, 12, 201-206 (2000).

27) Amakura Y., Tsutsumi T., Sasaki K., Yoshida T., Maitani T., Biol. Pharm. Bull., 26, 1754-1760 (2003).

28) Casper R. F., Quesne M., Rogers I. M., Shirota T., Jolivet A., Milgrom E., Savouret J. F., Mol. Pharmacol., 56, 784-790 (1999). 